\title{
Sampling by Fluidics and Microfluidics
}

V. Tesař

Selecting one from several available fluid samples is a procedure often performed especially in chemical engineering. It is usually done by an array of valves sequentially opened and closed. Not generally known is an advantageous alternative: fluidic sampling units without moving parts. In the absence of complete pipe closure, cross-contamination between samples cannot be ruled out. This is eliminated by arranging for small protective flows that clear the cavities and remove any contaminated fluid. Although this complicates the overall circuit layout, fluidic sampling units with these "guard" flows were successfully built and tested. Recent interest in microchemistry leads to additional problems due very low operating Reynolds numbers. This necessitated the design of microfluidic sampling units based on new operating principles.

Keywords: sampling, fluidics, microfluidics, fluidic valves, flow switching, fluid samples.

\section{Introduction}

Analysis of the chemical composition of fluids is an important operation in chemical engineering, often performed continuously, especially in monitoring the operation of chemical reactors. Requirements of accuracy and reproducibility make composition analysers expensive. When monitoring several reactors operating in parallel - with fluid composition varying slowly, as is often the case - it may be a good idea to use a single analyser sequentially evaluating samples taken from several locations. This requires placing a selector (or multiplexer) sampling unit in front of the analyser. This unit operates in discrete time steps: one sample is selected and delivered to the analyser at each time step. There are many possible variants. The fluid flows not selected at a particular instant may be halted (turning-down action), or dumped to a vent outlet (diverting action). The individual time steps need not be of the same duration. The sequence of the samples at the output may be simply repetitive periodic or varied according to some programme.

There are, nevertheless, features common to all sampling unit designs:

1) The unit has several input terminals and a single output (an exception being aggregate designs with, e.g., several outputs serving two or more analysers, etc.).

2) The unit performs a spatio/temporal conversion (Fig. 1).

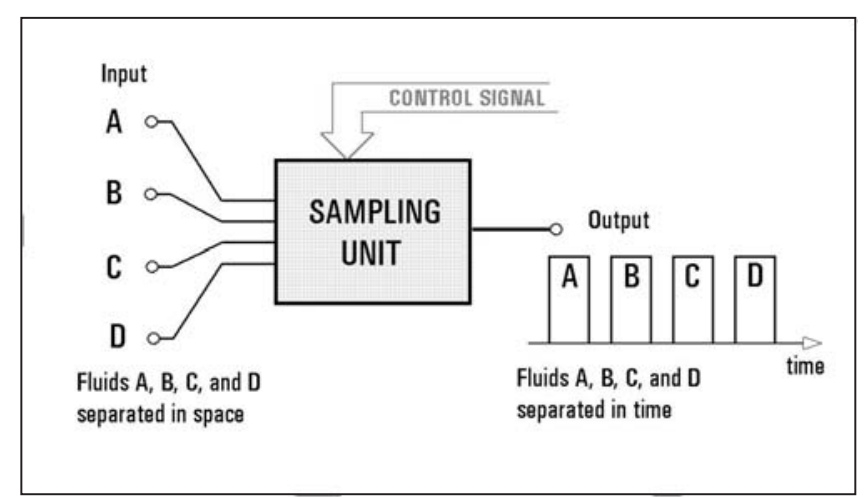

Fig. 1: The task of the selector sampling unit: converting spatial separation between fluid samples at the input into temporal separation at the output.
3) The operation is controlled by an external signal (though the signal generator may be integrated into the unit even to the degree of sharing some of its components).

4) An essential requirement is to eliminate any possibility of cross-contamination between the samples.

\section{Sampling reaction products from parallel chemical reactors}

Conventional sampling units use mechanical valves a single special multiposition scanning valve, or an array of common two-position valves. The schematic Fig. 2 shows

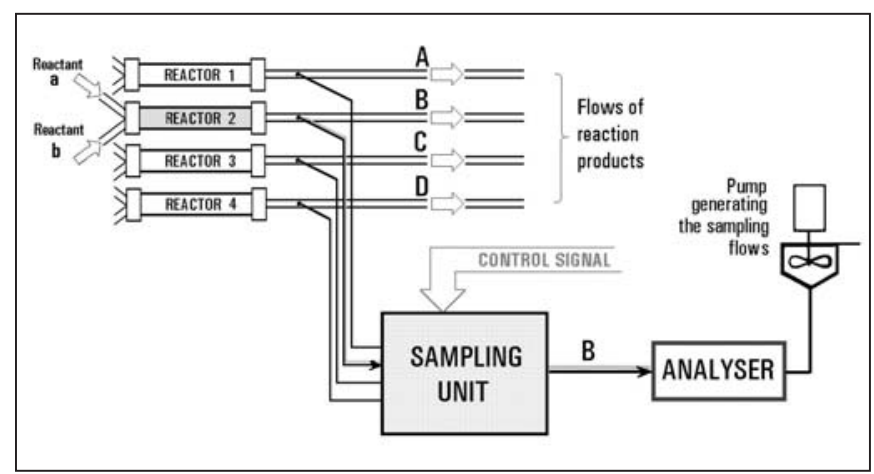

Fig. 2: Schematic diagram of a typical application of the selector sampling unit for testing reaction products at outputs of chemical reactors operated in parallel and sharing a single analyser.

a typical case. A modern trend in chemistry is to perform chemical processes in microreactors [3]. The required total production rate is obtained by "numbering up" - operating a large number of microreactors - instead of scaling up. One advantage of this approach is better controllability of the process, due to the substantially increased surface-to-mass ratio in small reactors. To use this advantage, the control system needs knowledge about the process. The composition of the reaction products is one of the principal items of information required. The arrangement according to Fig. 2 - often with hundreds of sampled reactors (rather than only four as shown here for simplicity) - has become increasingly common. 
Fig. 3 presents a standard version of the selector sampling unit, using two-positional mechanical valves. There are two essential constituent parts: the array of valves and the flow

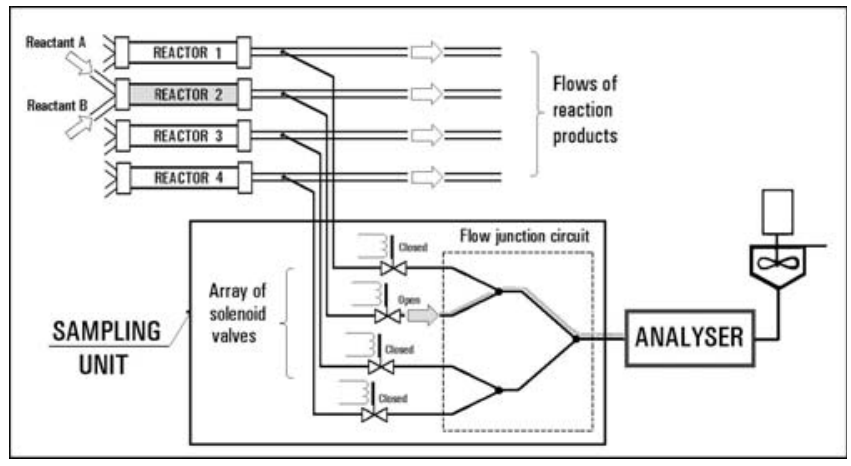

Fig. 3: Schematic diagram of the sampling unit from Fig. 2 with traditional control of the sample flows by solenoid valves. In this case the junction circuit may be simply a common connection of all pipes, as mechanically closed valves permit no flow in their "CLOSED" state.

junction circuit. The latter is admittedly trivial in the case of the mechanically closures of the flowpath in Fig. 3, where all valve outlets may be simply connected together.

\section{Fluidics: No-moving-part valves}

Chemical reactors commonly operate at high temperature and/or with aggressive fluids. This makes the conditions for the valves rather demanding. The small size of microreactors, calling for correspondingly small valves, makes the demands even more formidable. Although microvalves with moving parts are demonstrated, they are difficult to manufacture, expensive, and prone to breakages.

There are many advantages offered by an alternative: using purely fluidic valves [2]. These are easy to manufacture by methods used in micro-engineering, may be made from heat- and corrosion resistant material and have an almost unlimited operating life, even under extreme conditions. There are many variants - Fig. 4 presents what may be a typical example of a valve operating at large Reynolds numbers. This is a diverter type valve. The sample flow is accelerated in the main nozzle and directed as a jet towards the two opposite collectors (receivers). One of these is connected to the analyser output. The jet is deflected into it in the "OPEN" tate of the valve. The other collector is connected with the vent, into which the sample flow is dumped in the "CLOSED" state. The valve in Fig. 4 is symmetric, the roles of the two collectors are mutually interchangeable. Using the Coanda effect saves the power required for jet deflection. Just short switching pulses suffice for control - to move the jet from one attachment wall to the opposite one.

A valve is basically just a shaped constant-depth cavity, easily made by standard microengineering manufacturing techniques, such as etching. There is no expensive assembly of moving components, nothing to break or seize. It may well operate without maintenance at extremely high temperature.

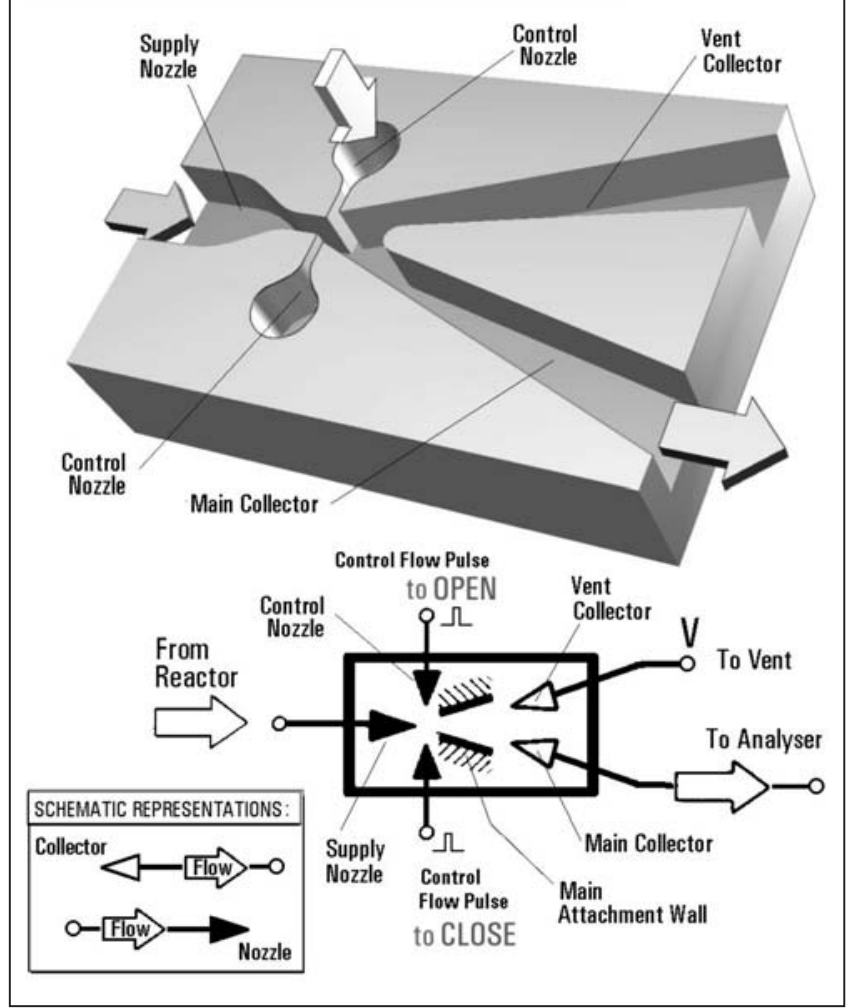

Fig. 4: An example of a typical no-moving-part fluidic valve. This is a jet-type bistable diverter, its bistability achieved by the Coanda-effect [7] attachment of the jet (issuing from the supply nozzle) to one of the two attachment walls. Black triangles in the schematic representation: nozzles. White triangles: collectors with their diffusers.

It is easily made even from refractory materials with high corrosion and abrasion resistance.

On the other hand, the design of a sampling unit with fluidic valves is quite exacting, much more than with mechanical valves and there are several inherent problems. The control signal is usually carried by a control fluid, which in some valve designs may mix with the sample fluid. Even the use of an inert control fluid, not detected by the analyser, does not avoid problems caused by this mixing: the sample is diluted, demanding higher analyser sensitivity. The fact that the sample flow is not turned down but diverted into the vent means the unit is complicated by the presence of vent channels. The greatest problem is caused by the complex hydrodynamic properties of fluidic valves when compared with their mechanical counterparts. When the solenoid valves in Fig. 3 are closed, no flow can pass through them, whatever conditions there are either upstream or downstream. This is not so simple in the case of a fluidic valve. When nominally in its "CLOSED" state - i.e., diverting the sample into the vent - the valve may either overspill some sample fluid into the analyser or, on the other hand, may return the fluid back from the analyser due to the jet pumping effect. Which of these two effects takes place depends upon the pressure levels in the other parts of the fluidic system. When designing a fluidic sampling unit, the pressure levels in the outlets must be carefully adjusted taking into account the valve loading characteristics. 


\section{Auxiliary Small Flows: Cavity cleaning flows and "guard" flows}

Overspilling into the analyser terminal in the "CLOSED" state is unacceptable. The overspilled fluid would contaminate the tested sample. On the other hand, the jet pumping effect (due to the entrainment into the jet) in the "OPEN" state leads to generation of reverse flow in the "off" outlet, which may be useful. Note in Fig. 3 that the channels and all other "dead volumes" downstream from the closed valves remain full of fluids different from the sample we wish to

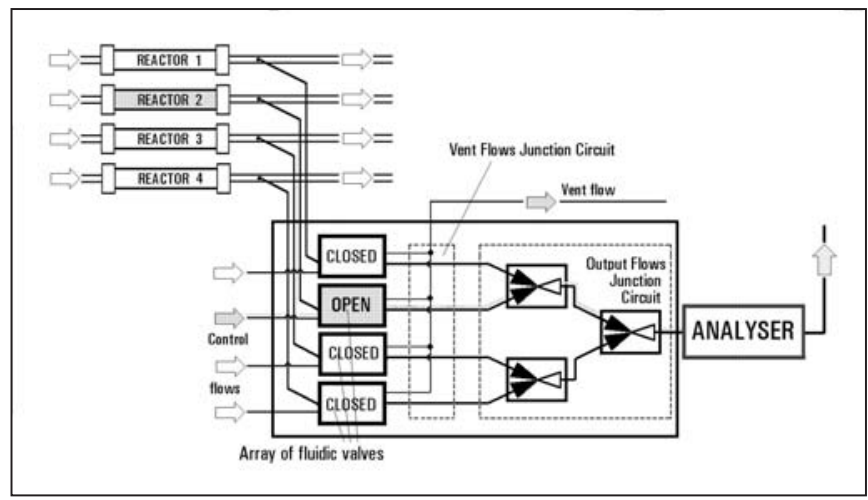

Fig. 5: Schematic diagram of the sampling unit from Fig. 3 with the solenoid valves replaced by fluidic diverters. Apart from the additional complexity of the control and vent channels, a more complex flow junction circuit is often required - in this example using symmetric jet pumps (Fig. 9).

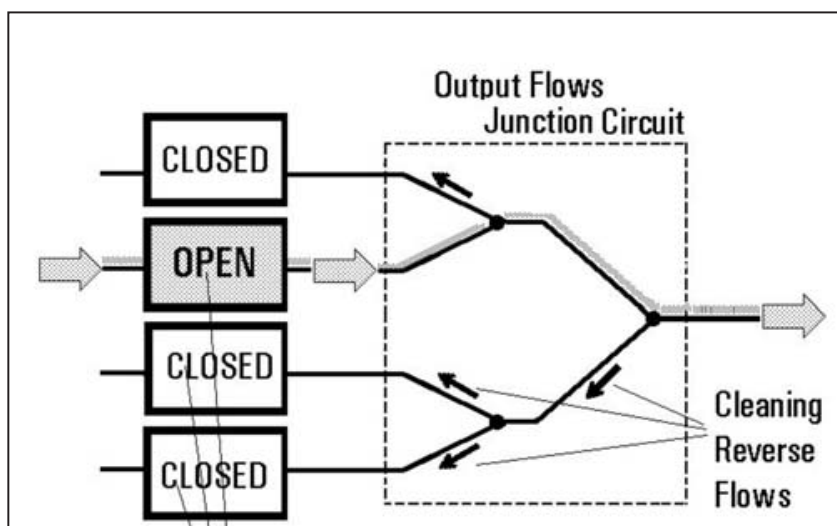

Array of fluidic valves

Fig. 6: Schematic representation of flows in the flow junction circuit. To eliminate sample cross-contamination, it is advisable to generate small reverse protective flows that clean the previous sample fluids from the downstream cavities.

deliver to the analyser. Of course, this fluid is stagnant, but it is impossible to ensure absolutely that it does not mix with the tested sample by diffusive and even eddy convection processes. As shown in Fig. 6, small reverse flows back into the "CLOSED" valves due to the jet pumping effect remove the contaminating fluid from these volumes - and thus assure high sample purity.
This capability to generate cleaning reverse flows is another important advantage of purely fluidic sampling units. Of course, reverse flows mean the loss of a part of the sample flow. To keep the loss acceptable, these cleaning flows must be very small.

The consequences of auxiliary protective flows for fluidic circuit design are shown here in examples using another, monostable version of fluidic valves, as shown in Figs. 7 and 8. Monostability is achieved by removing one of the attachment

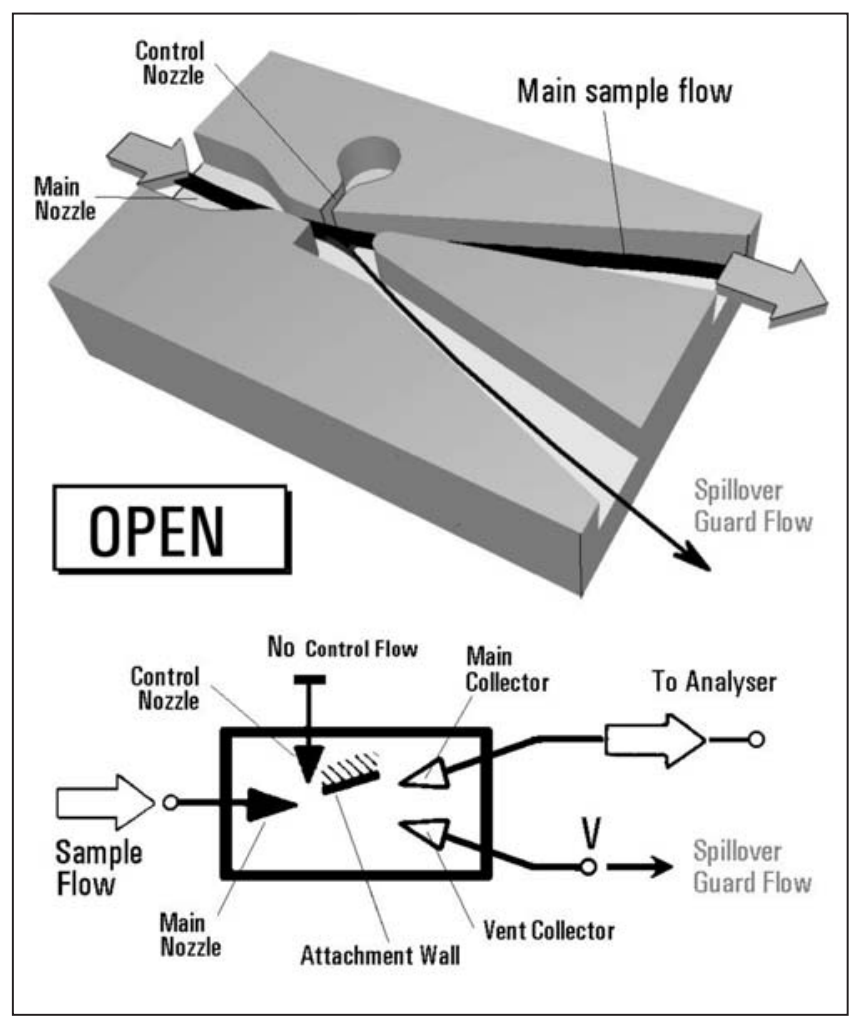

Fig. 7: An example of a monostable fluidic jet-deflection diverter valve, shown here in its "OPEN" state. There is only one attachment wall - the other wall on the opposite side is placed far from the main nozzle exit. As before, the black triangles in the schematic representation are nozzles, white triangles are collectors.

walls or placing it sufficiently far away from the nozzle exit to render the Coanda effect on this side ineffective. The advantage is simpler control, as there is just a single control channel per valve (note the two control channels per valve needed in Fig. 4). The disadvantage is the permanent control flow needed to keep the jet deflected (instead of just the short switching pulse needed by a bistable valve). However, with the small absolute values of flow rates in microchemistry, the control fluid consumption is usually not a very important consideration. Fig. 7 shows a monostable valve in the "OPEN" state, without control flow. The main jet always attaches to the remaining single attachment wall and is led into the analyser - without contamination or dilution by the control fluid. Fig. 8 shows the same valve with applied control flow, i.e., switched into the "CLOSED" state. The control fluid flow pushes the sample fluid jet away from the attachment wall and diverts it into the vent outlet. Contamination or dilution by the control fluid in this state is acceptable (it does not get to the analyser). The desirable backward flow through the main 


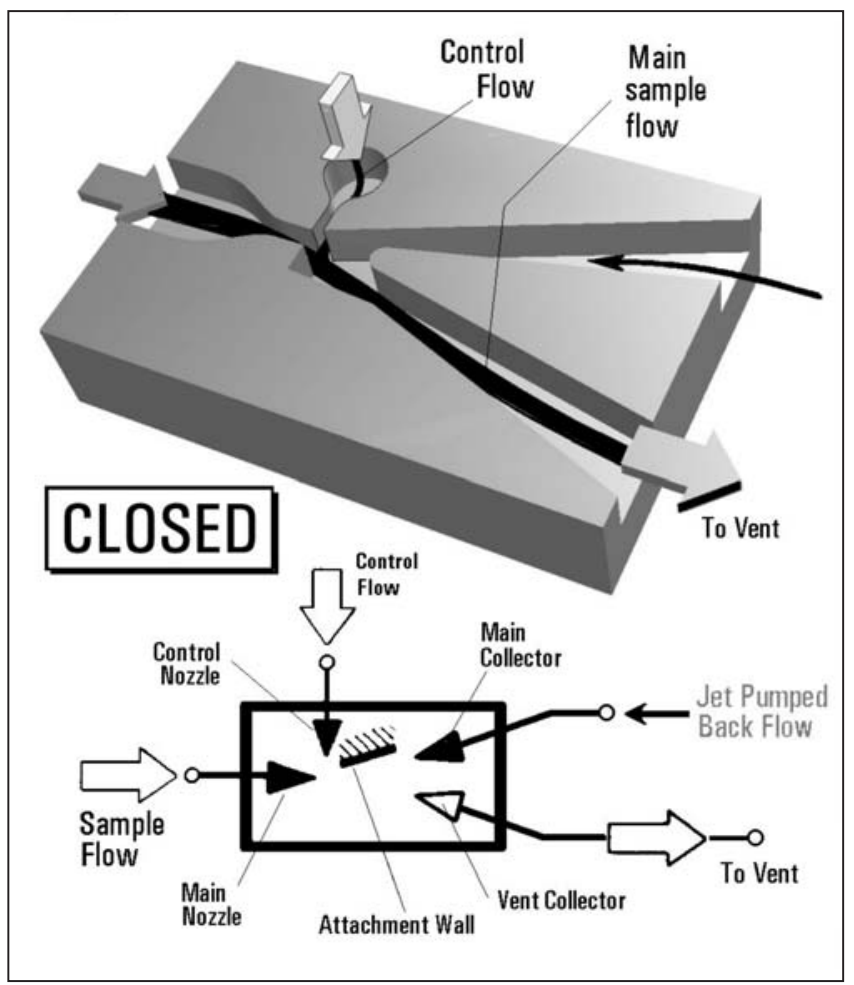

Fig. 8: The fluidic monostable diverter valve from Fig. 7 shown in its "CLOSED" state, with the desirable flow reversal in the main collector (which then temporarily operates as a nozzle).

collector - leading to the cleaning action according to Fig. 6 is due to the entrainment of outside fluid into the jet, similarly as in jet pumps.

The cavities through which the sample fluid passes in fluidic valves are not completely mutually separated - there are the common vent outlets through which they communicate. All but one sample flows and all control flows coming from the "CLOSED" valves meet there and freely mix. This "wild" mixture must be prevented from coming into contact with the sample passing through the only "OPEN" valve before it reaches the analyser. The sample purity requirements call for another small auxiliary protective flow to be generated. This "guard flow" is obtained by sacrificing a small amount from the sample flow and directing it into the vent as shown in Fig. 7. To generate the "guard flow" and the "cleaning reverse flow" in the "off" collectors in the two states of the same valve is not easy - due to the mutually opposite requirements. It is possible, but it calls for careful adjustment of the conditions.

\section{Flow Junction Circuits: Flow enhancing and flow inhibiting elements}

The task of getting the desired orientations of the small auxiliary flows in the "off" collectors of valves may be made easier and less dependent on precise adjustments by using additional fluidic elements placed into the junctions of the valve exits. This results in special flow junction circuits (cf. Fig. 5 and Fig. 6). The fluidic elements there are also no-moving-part devices (sharing the advantages of easy manufacture and extreme reliability under adverse conditions). Their task is to generate a specified mutual interaction of the flows that meet in them. The effects are dependent upon dynamic effects in flowing fluids, which again limits the use of these elements to higher Reynolds numbers, of the order of at least $10^{2}$.

The two basic cases of such fluidic flow interaction elements are shown in Fig. 9 and Fig. 10. In spite of their opposite roles, they are physically quite similar. Both consist of two nozzles and one collector and differ only in the magnitude of

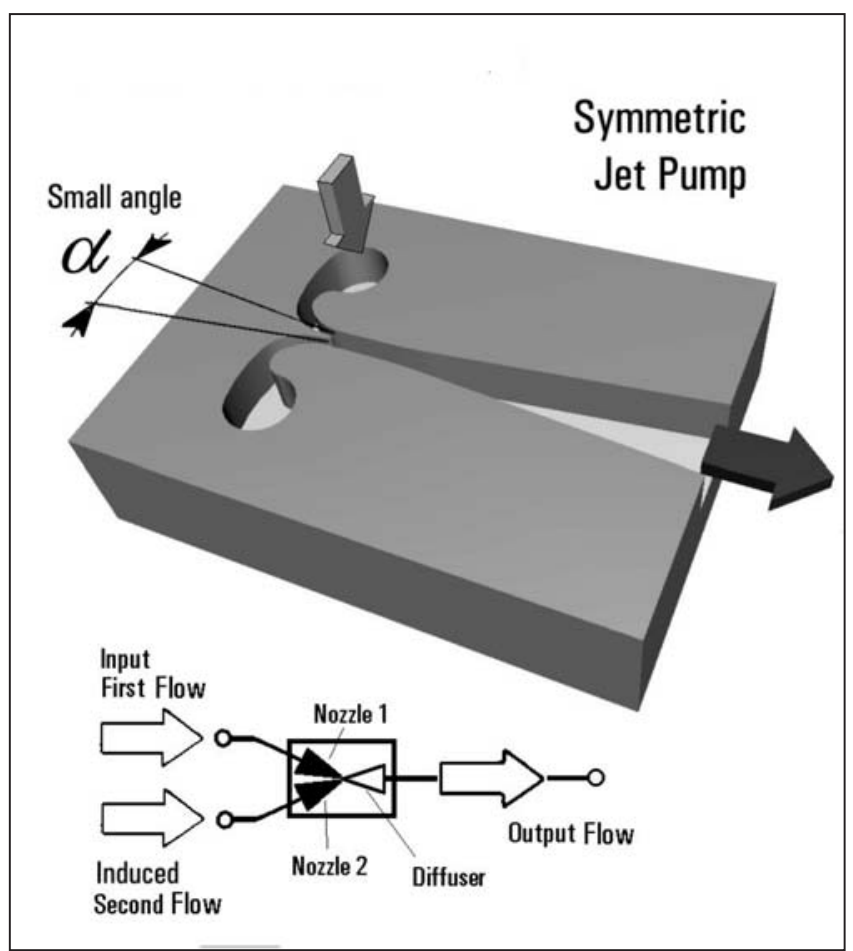

Fig. 9: Simple fluidic flow junction element - a symmetric jet pump - in which one of the incoming flows enhances the magnitude of the flow in the other inlet.

the angle at which the nozzle exits meet. If the angle is small, as in Fig. 9, the jet generated in one nozzle induces the flow in the other inlet by the jet pumping action. In fact, the element in Fig. 9 is a jet pump, differing from common jet pumps only in being symmetric, as the role of the two inlets must be mutually interchangeable - each can serve as either a driven or a driving inlet. The element is connected to the vents of two adjacent diverter valves to generate flows directed away from the valves. This is useful for promoting or even generating "guard" flows. The symmetry of the layout slightly compromises the achievable jet pumping effect - but this is not very important, since the required generated "guard" flows must be small anyway.

The very opposite effect is achieved in the other element shown in Fig. 10. It has a large angle $\alpha$, near to $\pi$. The flow admitted into one of the nozzles tends to generate a flow of opposite sign in the other inlet. This element is usefully connected to the output terminals of two adjacent diverter valves. The sample flow from the valve in its "OPEN" state 


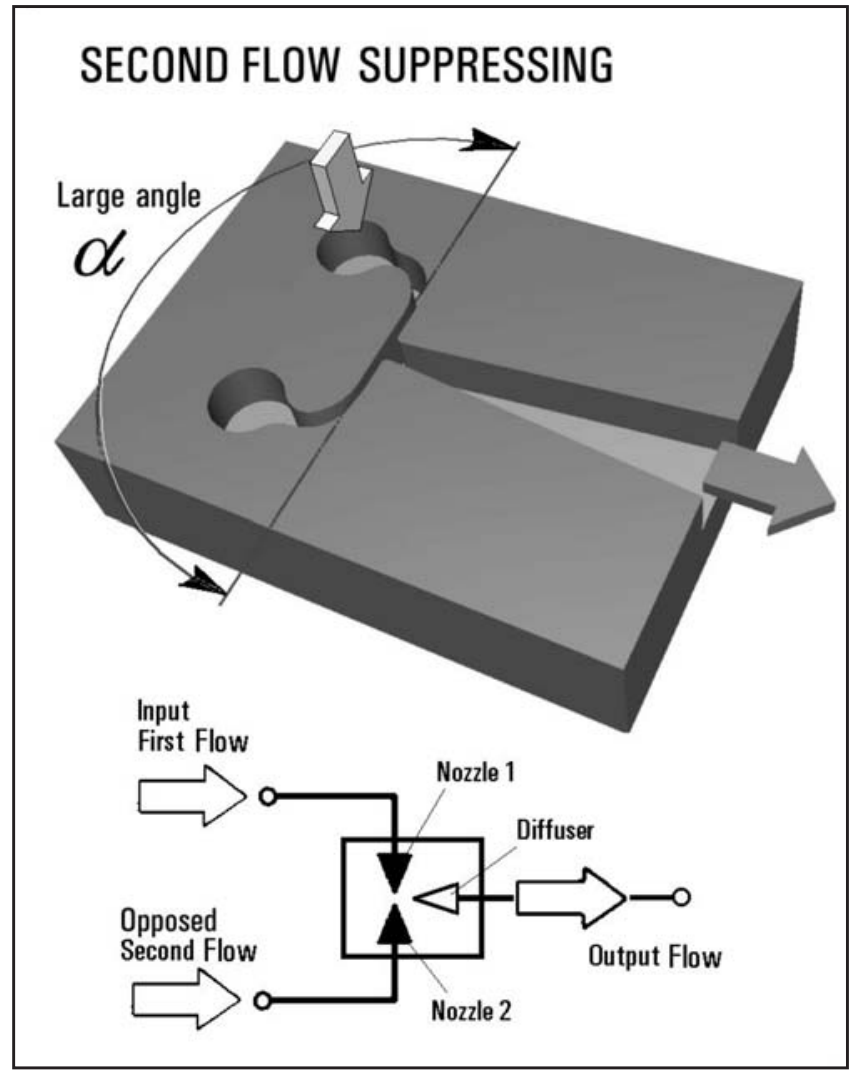

Fig. 10: Another simple fluidic flow junction element - differing from the one in Fig. 9 only in the mutual inclination angle $\alpha$ of the two nozzles. In this case one of the incoming flows suppresses or even reverses the flow in the other inlet.

generates the desirable backward flow through the output collector of the other valve, which is in the "CLOSED" state.

Of course, specific requirements of pressure and flow balancing in the junction circuit may call for devices in between, with intermediate values of the angle.

\section{Complete sampling unit}

A simple, two-valve case of a unit built from the elements described above is shown in the circuit diagram Fig. 11. It is switched between only two sample flows. Both flow interac-

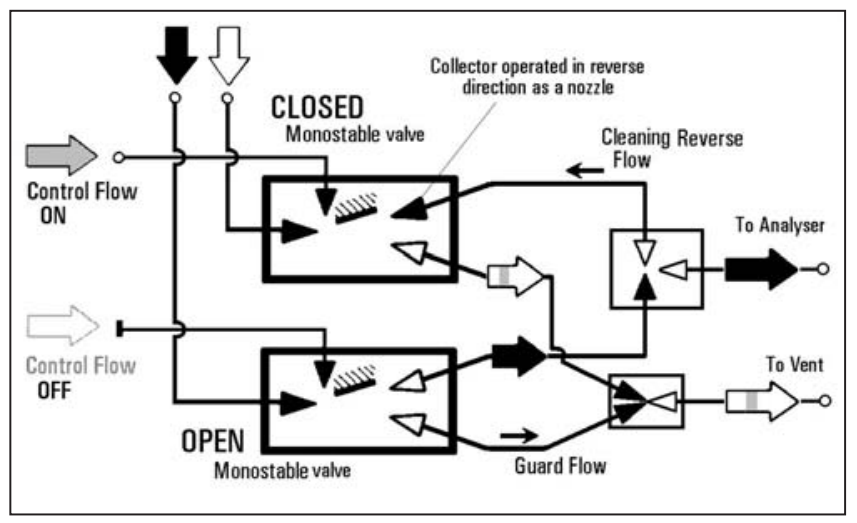

Fig. 11: Schematic diagram of the basic circuit of the fluidic sampling unit - with only two inlets and two monostable valves, one of them "OPEN" while the other is "CLOSED". tion elements as discussed above are used here in the valve outlets. The flow reversing element (Fig.10) is connected to the output terminals, its outlet leading to the analyser. The flow enhancing element (Fig. 9) is connected to the vent outlets of the valves. Such complete junction circuits - with both kinds of flow interaction elements - are, however, rare. At least one of the desirable small protective flows (and sometimes both) may be generated just by proper adjustment of the pressure levels.

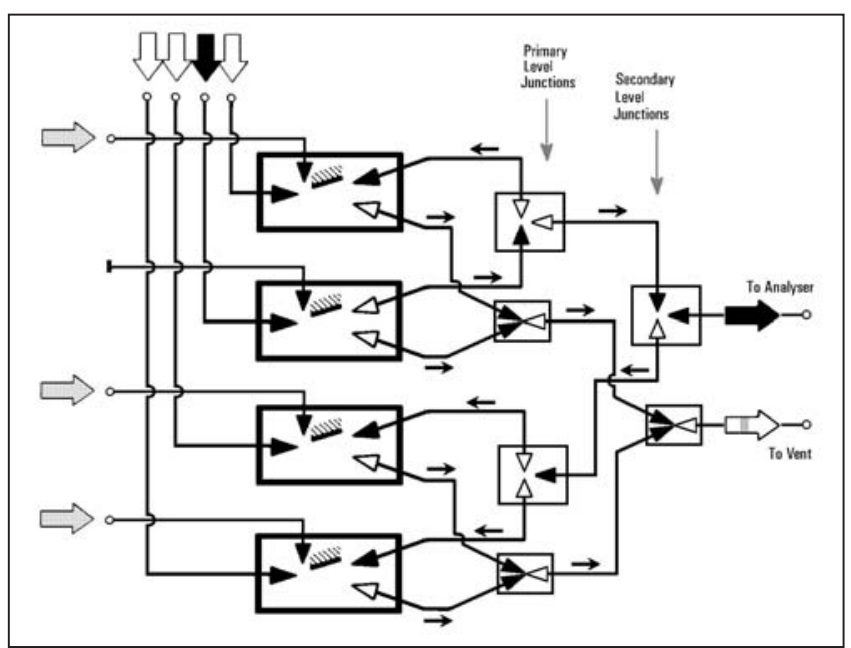

Fig. 12: Circuit diagram of a fluidic sampling unit with four inlets. The complexity of the circuits with the various directions of the flows (indicated by arrows) is increased, but the basic principle is a simple repetition of the connections from Fig. 11.

Fig. 12 extends the above example by presenting a sampling unit designed to sample four channels. There are two valve pairs from Fig. 11, each having its two flow interaction elements in the flow junctions at the primary level. The outlets from each pair are then connected to interaction elements of the secondary level junctions. A similar tree-like connection principle at higher levels is applied to build sampling units for a larger number of sample flows. The tree-like interconnections need not be completely symmetric (in the case of six sample flows the primary level junctions of the third switched valve pair are connected directly to the tertiary level interaction elements).

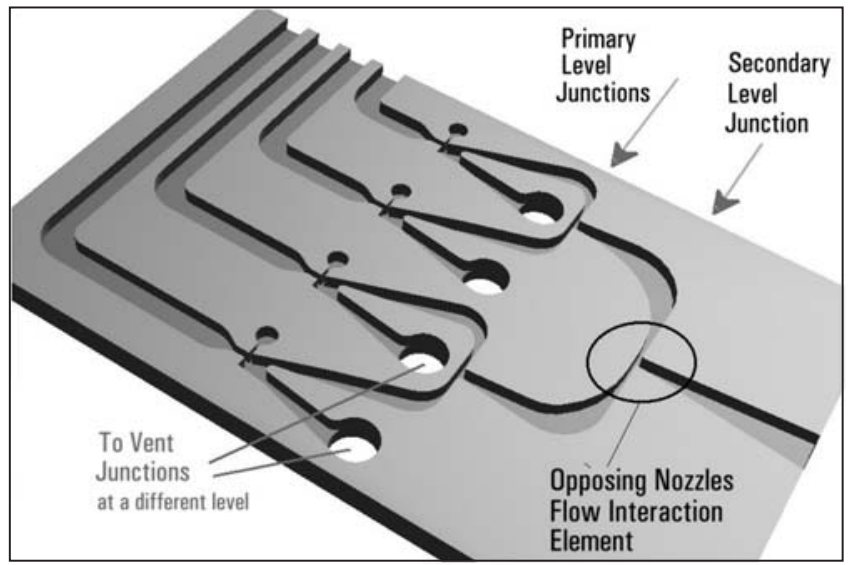

Fig. 13: Example of a fluidic sampling unit with four inlets, etched integrally in a single plate. 
The disadvantage of this layout is the inevitable crossing of the valve outlet channels. Manufacturing by etching all the valves and interconnecting channels in a single plate is thus impossible. In Fig. 13, showing the appearance of the corresponding sampling unit from Fig. 12, the flow enhancing junction elements are not visible, being located at another level, in a different plate stacked on top of the plate shown here.

\section{Low Re microfluidic sampling units}

The continuing tendency towards decreasing the device size has already led to microdevices of micrometer size, not visible to the naked eye. Typical present-day microreactors are larger, but some of them have been built in submillimetre dimensions. Typical applications are on the one hand in analytical chemistry - the "lab-on-chip" performing hundreds to thousands of parallel analyses, such as DNA "fingerprinting", which may make bring to reality the goal of objective diagnosis of diseases (another business behind the recent efforts is paternity testing). Another promising field is synthetic chemistry, currently aimed particularly at producing liquid fuels (and processing them for fuel cells).

At the submillimetre scale, the associated microfluidic valves required by microreactors - for reactant flow control as well as sampling units - need to operate at low Reynolds number Re, the product of flow velocity and dimension (usually the main nozzle width) divided by viscosity. Low Re sampling units are required to handle high viscosity fluid samples, such as viscous biological liquids or hot gas. The flow rates and hence velocities are quite often small, because the total flow through a reactor is limited by the requirement of reactor residence time - and the samples taken from them represent a loss missing from the production, so that there is every reason to keep them at a minimum. Reynolds number may be interpreted as the ratio of inertial forces to viscous forces acting on the fluid. If it is low, the dynamic effects even in an accelerated fluid downstream from a nozzle are weak, the flow is dominated by viscous damping. These inertial effects, however, are the very phenomena upon which the operation of fluidic valves and other elements as described above is based. Below about $\operatorname{Re}=40$, a flow issuing from a nozzle forms no jets at all. The fluid simply spreads equally in all available directions. In this subdynamic regime [4], the operation of jet-type fluidic elements ceases to be possible. In fact, the Coanda-effect attachment to a wall is absent already below about $\operatorname{Re}=800$.

This means that with the present tendency towards smaller and smaller size, fluidic sampling units as described above may increasingly often be found to be out of the question the microfluidics applications [4]. New operating principles are needed. Indeed, some recent microfluidic devices rely on unusual driving effects - such as electro-osmotic forces. It is, however, possible to remain within the domain of classical hydromechanics by using pressure driven valves. Instead of relying on the acceleration in a nozzle, the sample flow is forced through the valve by a constant pressure difference between vent $V$ and output terminal $Y$. Generating and maintaining this pressure difference may require the addition of a pressure regulator. This is usually no problem in the context of MEMS (micro electromechanical systems), of which micro- fluidic sampling units form a part - often made by etching directly on a silicon chip together with the electronic control circuits, which are thus readily available.

At low Re there is no point in using flow interaction elements (Fig. 9 and 10) - jet pumping fails without turbulence and flow collision ceases to be interesting without inertial effects. The terminals of all valves of the low-Re unit both vent terminals $V$ and output terminals $Y$ - are usually simply mutually connected. This means the driving pressure difference $\Delta P_{\mathrm{YV}}$ is simultaneously acting in all the valves in the unit. The driving pressure must be carefully adjusted so as to obtain in the "OPEN" state not only the required sample flow into the output terminal $Y$, but at the same time also a small but desirable spillover "guard flow" into the vent terminal $V$. The problem then arises in the "CLOSED" state. First, it is more difficult to generate the desirable cleaning back flow, since it must overcome the pressure difference $\Delta P_{\mathrm{YV}}$ acting in the opposing direction. At the same time, there is no hope of fulfilling this more difficult task by the jet pumping effect generated by the sample flow, which is too slow.

\section{Jet pumping by a powerful control flow}

One solution, covered by British Patent Applications [1], [5] was found by the author, and is described in ref. [4]. The idea is that if the sample flow is too weak, another available flow must be used - and the only flow that may be sufficiently powerful under the circumstances of the "OPEN" state is the acting control flow. This idea had to overcome a psychological obstacle: designers of fluidic valves assume quite naturally that the control flow is many times smaller than the controlled flow - this is why valves are usually called "fluidic amplifiers". The ratio of the supply flow rate to the control flow rate, usually made as large as possible, is called the "flow gain". The new idea had to accept disproportionately large control flows and hence extremely low, in fact fractional, values of the gain.

As shown in Figs. 14 and 15, the new microfluidic valves incorporate a classical, large Re fluidic element: a jet pump. This generates the desired cleaning return flow in the "CLOSED" state, Fig. 14. The exit flow from the jet pump is

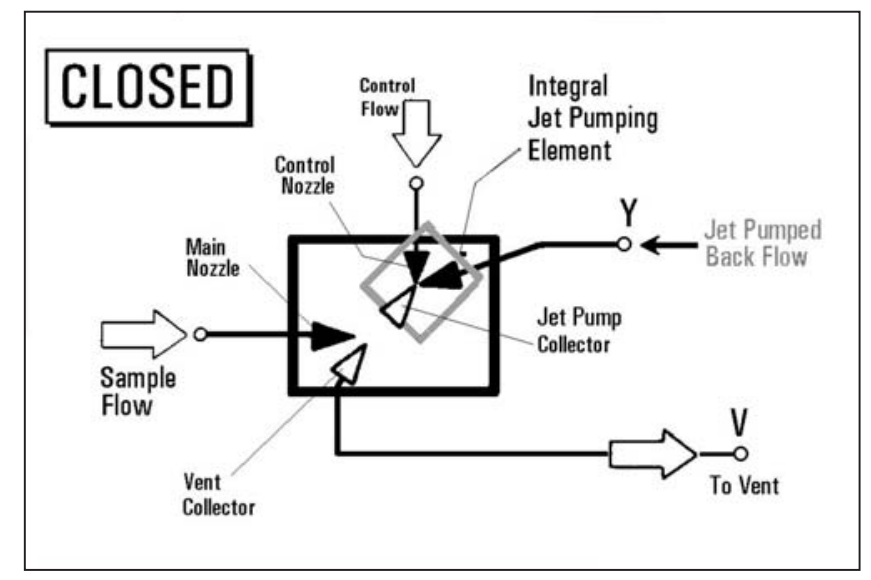

Fig. 14: Schematic representation of a fluidic valve [5] generating the desirable reverse cleaning flow in the "CLOSED" state by the jet pumping effect by a powerful control jet. For this purpose, the valve contains what is basically an integral jet pump. 
directed towards the vent collector. This is placed on the opposite side of the sample flow path, so that the powerful jet pump exit flow sweeps the sample into the vent as well. This is the principle of the closing action. In Fig. 14, the axes of the jet pump and vent collector are inclined with respect to the main (sample supply) nozzle axis. This is a useful feature, since jet momentum interaction studies indicate that optimum jet deflection is achieved with the control jet inclined to have a velocity component opposed to the supply flow velocity. The inclination is even more useful in the "OPEN" state valve state (Fig. 15). The sample flow has to pass through the valve into the output terminal $Y$, and it is advantageous to have the path towards this terminal as straight as possible to get a lower value of the pressure drop $\Delta P_{\mathrm{YV}}$

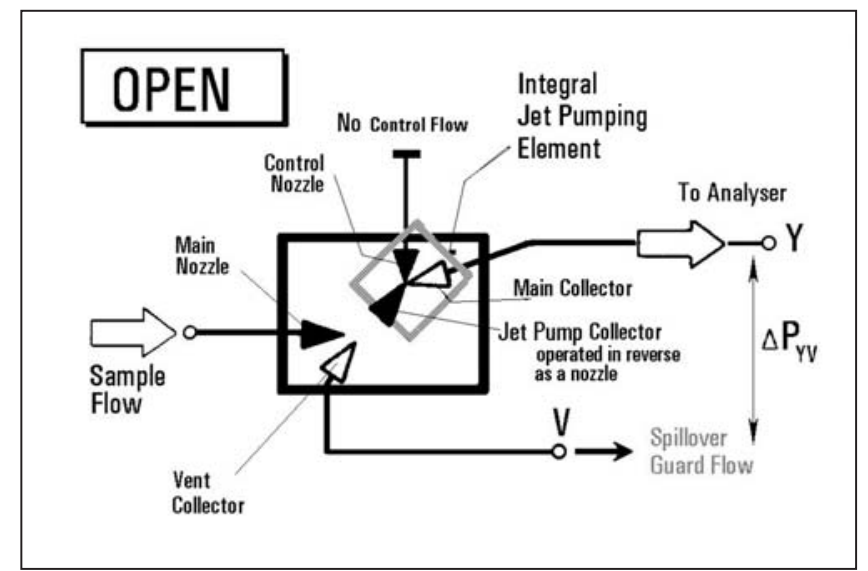

Fig. 15: The fluidic valve from Fig. 14 shown schematically in its "OPEN" state. The flow to the analyser is driven by the applied constant pressure difference $\Delta P_{\mathrm{YV}}$, adjusted so as to obtain the proper magnitude of the "guard flow".

\section{Microfluidic valve and an example of the sampling unit}

Designing a microfluidic valve based on this principle is a question of finding the best compromise between the conflicting requirements of the "CLOSED" state Fig. 14 and the "OPEN" state Fig. 15. An efficient layout of the jet pump part - e.g., according to Fig. 19 - improves the achievable clearing backflow in the "CLOSED" state, but because of the ensuing complexity of the sample path, this means a higher pressure loss $\Delta P_{\mathrm{YV}}$ in the "OPEN" state to be maintained by the pressure regulator - and to be overcome by stronger "CLOSED" state jet pumping.

In the example shown in Fig. 16, no attempt is made to have a really efficient jet pump and there is only a very short jet-pump collector (cf. Fig.14). The idea is to make the flow path through the "OPEN" valve shorter and easier, generating a lower pressure loss $\Delta P_{\mathrm{YV}}$. This means a lower pressure difference to be overcome by jet pumping, which therefore can afford to be less effective. Another feature typical for low-Re microfluidic devices is the missing cross-sectional area contraction in the supply ("main") nozzle, as there is no point in trying to accelerate the sample flow there.

The operation of a sampling unit using these valves may be followed in the circuit diagram of the basic valve pair shown in Fig.17. To recognise the degenerated character of the jet-pump collectors in these valves, the collector symbols

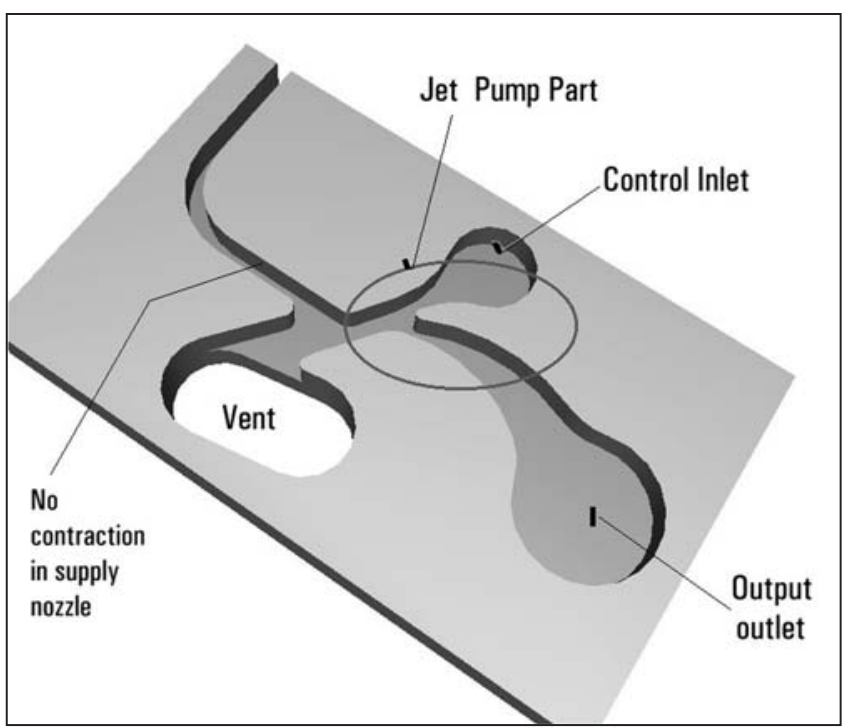

Fig. 16: An example of a microfluidic valve [5] for low-Re microfluidic sampling units. The desired reverse flow in the "CLOSED" state is obtained by the jet pumping effect of the control flow (cf Fig. 14). The jet pump is very rudimentary. The actual size is at least by an order of magnitude smaller than for the fluidic valves in Fig. 8.

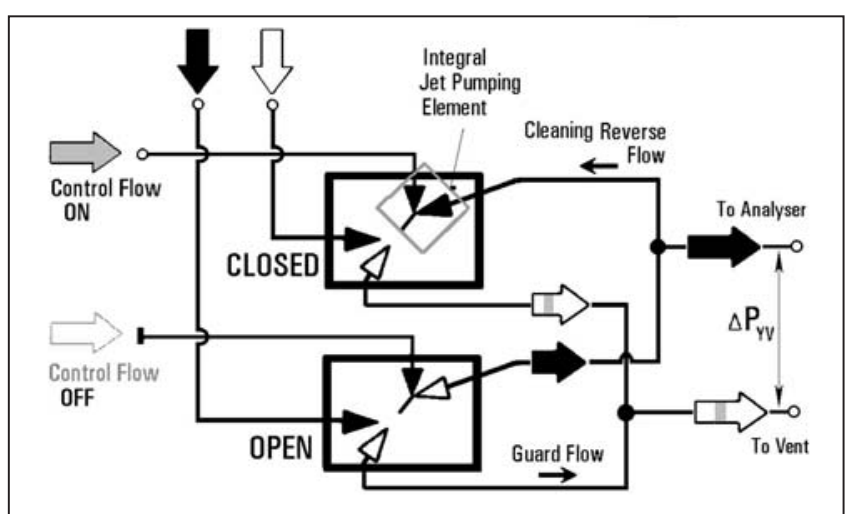

Fig. 17: Schematic circuit diagram of the basic valve pair unit (cf. Fig. 11) which forms the basis of microfluidic sampling units [1] - here with the valves from Fig. 14. The constant driving pressure difference $\Delta P_{\mathrm{YV}}$ is applied between the exit terminals.

in this schematic diagram are replaced by just a symbol of a short channel. There are no flow interaction fluidic elements in the junctions of the valve exits. As a consequence the keeping of the driving pressure difference $\Delta P_{\mathrm{YV}}$ is simplified - it has to be kept just between the final exit terminals of the complete sampling unit circuit. This would, of course, also apply if there were more valve pairs in a sampling unit handling a larger number of sample flows.

\section{Second example of a microfluidic valve and sampling unit}

In the other example of a microfluidic valve, shown in Fig. 18, the idea of limited jet pumping efficiency traded-off for smaller driving pressure difference $\Delta P_{\mathrm{YV}}$ was taken even further - to the degree of the jet pump being hardly recognisable. The sample flow path in the "OPEN" state is here only slightly curved. The rudimentary jet pump suffices since the 


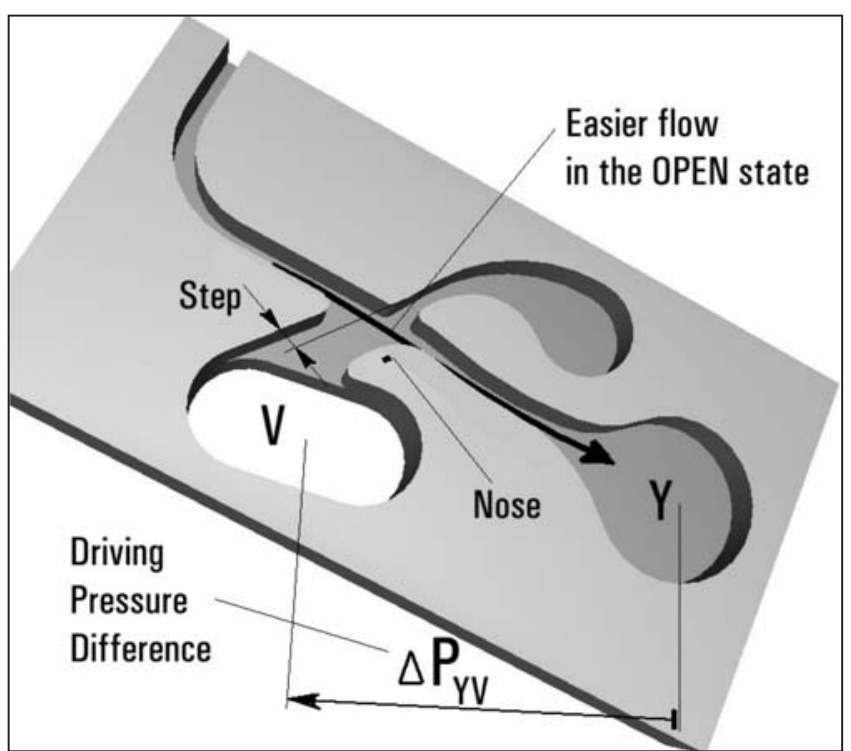

Fig. 18: Another example of a microfluidic valve based upon the principles from Fig. 14, with an even more rudimentary jet pump part. The advantage is its less tortuous flow path in the "OPEN" state, leading to smaller driving pressure difference $\Delta P_{\mathrm{YV}}$.

cleaning back flow is to be very small. This is due to the large number of valves in a realistic sampling unit, all of them simultaneously in their "CLOSED" state - while only one valve is "OPEN". If the back flow is only $1 \%$ of the sample flow, then with 80 valves generating the jet pumping back

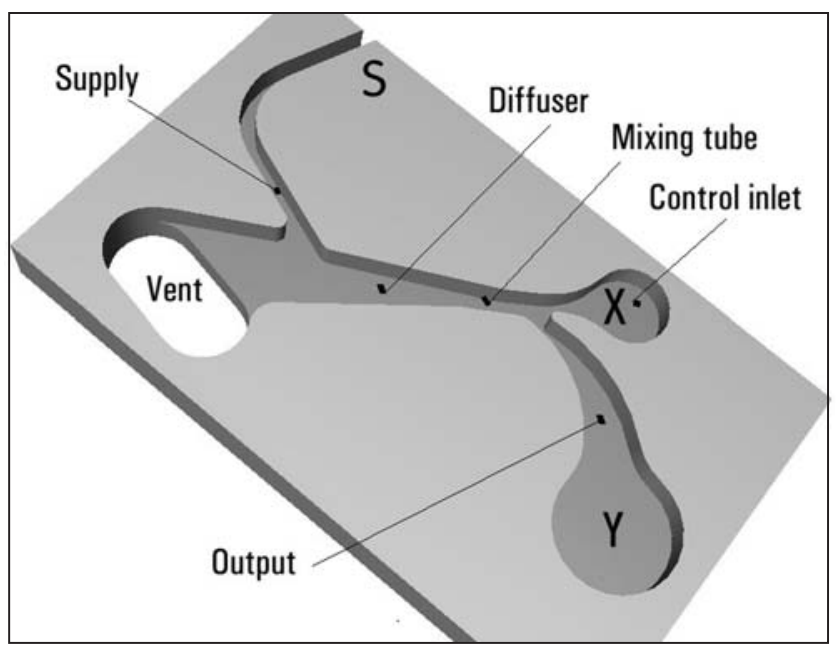

Fig. 19: Another example of a microfluidic valve based on the same principle as in Fig. 14 - this time with a full jet pump: note the long mixing tube and the even longer diffuser.

flow the total loss is $80 \%$ of the sample.

An important feature of the design is the "Nose" (Fig. 18) that seals off the output channel from the vent area and prevents the generated low pressure from being equalised by unwanted return flow from the vent space. The computed total pressure field in Fig. 20 (three-dimensional FLUENT solution using a two-dimensional turbulence model with RNG low-Re-turbulence modification) documents that even the reduced ,jet pump“ generates sufficient jet pumping. Note in Fig. 18 or Fig. 20 that there is no such "Nose" on the opposite, supply nozzle side. There is, on the contrary,

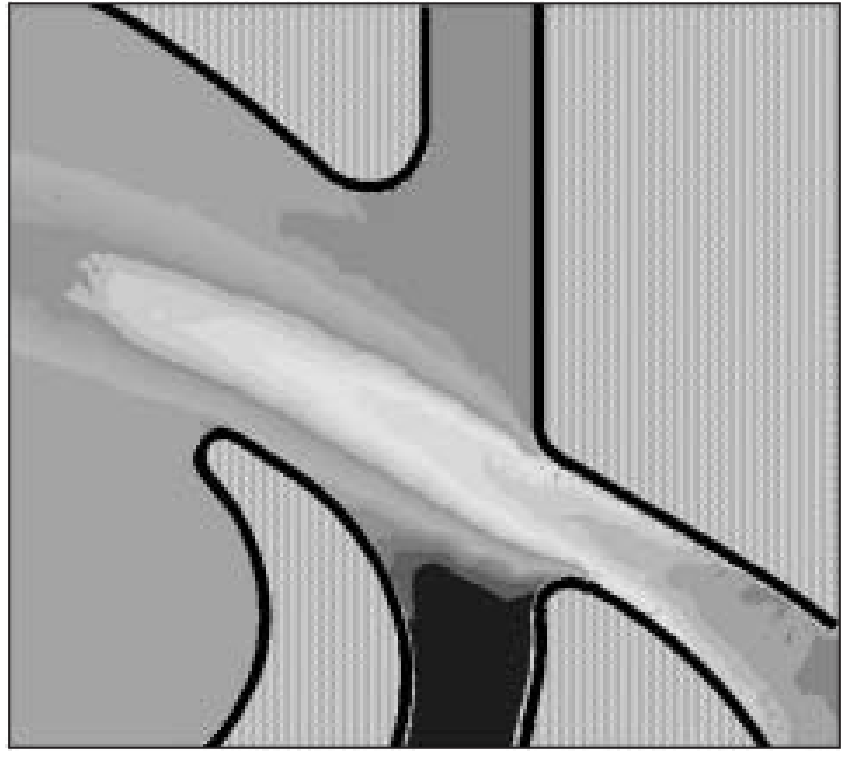

Fig. 20: Computed dynamic pressure field in the valve from Fig. 18 in the "CLOSED" state. Decreasing pressure values coded by increased darkness of the grey scale. Note that even rudimentary "jet pump" is capable of generating quite low pressure (very dark grey colour) in the output collector, provided the control jet is really powerful.

a setback "Step". Its task is to reduce the effect of the powerful control flow on the sample flow. Even though we talk here about a "CLOSED" state, it is undesirable to limit or otherwise influence the sample flow, as this would lead to unwanted changes in the reactor operation.

The valve example from Fig. 18 was tested by the author in a sampling unit [6] built for a research facility with a relatively small number - 16 - test reactors for high-throughput testing of catalysts for the Fritsch-Tropfsch reaction (hydrogenation of carbon monoxide to ethanol). Since this is a high-temperature reaction $\left(400^{\circ} \mathrm{C}\right)$, and the sampling unit is located in the vicinity of the reactors, it was manufactured in stainless steel and its control is external. The valves are switched by essentially cold nitrogen control flows. Fig. 22 shows the complete sampling unit made by etching in a $0.25 \mathrm{~mm}$ thin stainless steel foil, together with the flow-equalising restrictors. To achieve a higher aspect ratio of

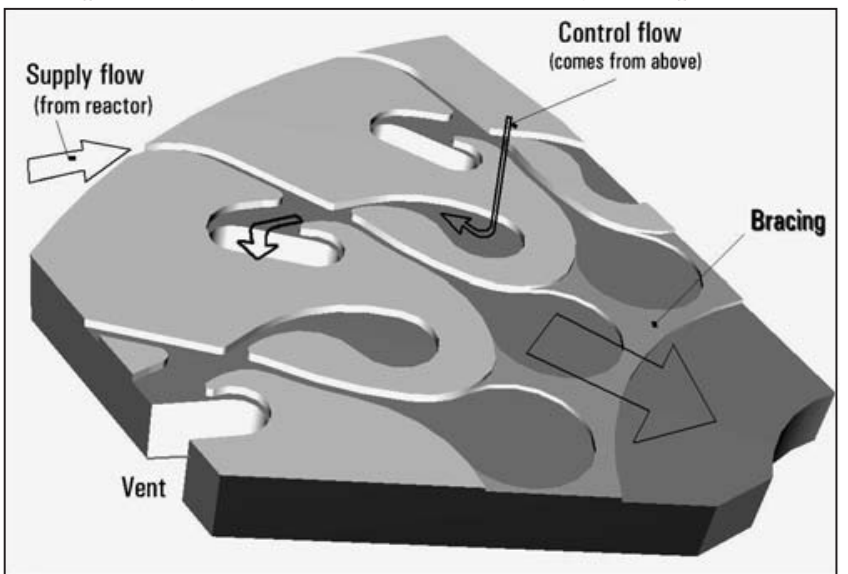

Fig. 21: A detail of a microfluidic sampling unit [1], [5] for high temperature applications, with valves according to Fig. 18 arranged into a radial array. The unit is made by etching in a stainless steel foil. 


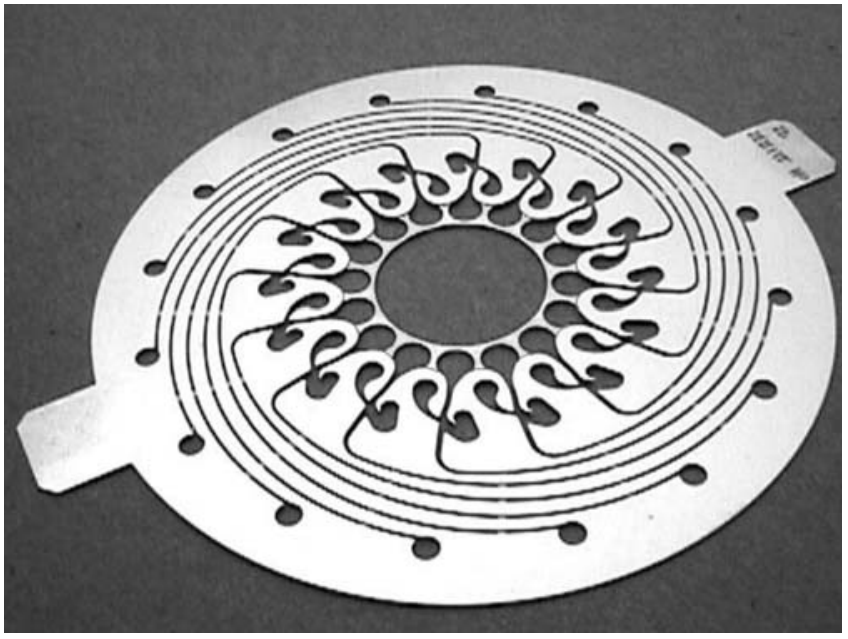

Fig. 22: Photograph of the author's microfluidic sampling unit [1], [4] with 16 pressure driven valves and their upstream restrictors (the spiral-shaped channels). The unit is made by through (two-sided) etching in a $0.25 \mathrm{~mm}$ thin stainless steel foil.

the cavities, two-sided etching was used. This would separate the internal part of the foil into 16 mutually movable parts. Structural integrity is obtained by braces (seen in Fig. 21) located downstream from the valves. The braces are etched only from one side so that there is a space above them allowing sample flow into the central cavity, which is connected to the analyser.

The valves are not extremely small - the supply nozzle channel width is $0.34 \mathrm{~mm}$ - but they operate at very low Re. The sample fluid is "syngas" - fuel synthetisation gas, a mixture of hydrogen and carbon monoxide. It reaches the valve at pressure $0.5 \mathrm{MPa}$ (a substantial decrease from $4 \mathrm{MPa}$ in the reactors). Despite cooling of the valve plate by the nitrogen control flows, the kinematic viscosity of the sample gas (also due to its large contents of $\mathrm{H}_{2}$ ) in the supply nozzle is as high as $40 \cdot 10^{-6} \mathrm{~m}^{2} / \mathrm{s}$. The nozzle exit bulk velocity is only about $3.8 \mathrm{~m} / \mathrm{s}$ - despite the exceptionally favourable circumstance of the reactors serving only for tests, so that full reaction product flow is available for composition analysis. As a result, the design had to cope with a very low supply nozzle Reynolds number, about $\mathrm{Re}=32$. The "guard flow" magnitude is adjusted by the choice of driving pressure, kept by an external pressure regulator, so that $6 \%$ of the sample flow in the "OPEN" state is spilled over into the vent. Only $3 \%$ return flow in the "CLOSED" state is chosen (which still means sacrificing in total more than one half of the available sample). In spite of this small desired return flow, the necessary control flow rate to generate the jet-pumping effect is about 40 times the sample mass flow rate supplied into the valve (in standard fluidics this would be an absurdly small flow gain of 0.025). With lower viscosity of the control nitrogen gas and a narrower, $0.27 \mathrm{~mm}$ wide, control nozzle to get higher control jet velocity (around $55 \mathrm{~m} / \mathrm{s}$ ), the Reynolds number of the control jet is around $\mathrm{Re}=1000$, just high enough to get at least some vortex entrainment effect.

\section{Conclusions}

This paper presents essential information about a little known way - perhaps more difficult to design, but bringing interesting advantages - to connect an analyser sequentially to one fluid source at a time from a number of available sample sources. As long as the source sample flow can achieve a Reynolds number of at least $\mathrm{Re}=200$ in the nozzle of the fluidic valve (and preferably more), it is possible to utilise what is essentially an already existing technology of fluidic jet-type valves and flow interaction elements. The complicating factor is the requirement to generate protective small flows to keep perfect sample purity. This requirement becomes a real problem in present day microfluidics, characterised by the tendency to operate at very low Re values. A solution based on the idea of an exceptionally powerful control jet was demonstrated to work successfully in a sampling unit built for operating with high-temperature gas.

\section{Acknowlegments}

The development of the microfluidic sampling unit for hot synthetic gas was supported by iAc (Institute of Applied Catalysis, London, U.K.) as a part of the high throughput catalyst testing project managed by Prof. R. W. K. Allen. The author has gained much useful information from discussions with Y. Y. Low and particularly with Dr. J. R. Tippetts, who is the originator of the earliest ideas on fluidic sampling.

\section{References}

[1] Tesař, V., Tippetts, J. R. T., Allen R. W. K.: Fluid Multiplexer. British Patent Application GB 0019767.9, April 2000.

[2] Tesař, V.: Valvole fluidiche senza parti mobili. [in Italian] Oleodinamica - pneumatica, revista delle applicazioni fluidodinamiche e controllo del sistemi. 1998, Vol. 39, No. 3 , p. 216. ISSN 1 122-5017.

[3] Ehrfeld, W.: Microreaction Technology: Industrial Prospects. Berlin: Springer, 2000, ISBN 3-540-66964-7.

[4] Tesař, V.: Microfluidic Valves for Flow Control at Low Reynolds Numbers. Journal of Visualisation. Tokyo: 2001, Vol. 4, No. 1, p. 51-60. ISSN 1343-8875.

[5] Tesař, V., Tippetts, J. R. T., Allen, R. W. K.: Fluidic Valve. British Patent Application GB 0003969, March 2000.

[6] Tesař, V., Allen, R. W. K., Tippetts, J. R, Low, Y. Y., Adams, C.: High Throughput Catalyst Testing: a Novel Multichannel Microreactor with Microfluidic Flow Control System. $8^{\text {th }}$ NICE (Network for Industrial Catalysis in Europe) Workshop on Fast Analytical Screening of Catalyst and Fast Catalyst Testing. Espoo (Finland): September 2000.

[7] Tesař, V., Randa, Z.: Large Attachment-Wall Divergence Angles in Fluidic Bistable Coanda-Effect Diverters. Proc. of Colloquium "Dynamika Tekutin '93", Inst. of Thermomechanics, AS CR Praha, October 1993.

Prof. Ing. Václav Tesař, CSc.

phone: 4401142227551

fax: 4401142227501

e-mail: v.tesar@sheffield.ac.uk

Department of Chemical and Process Engineering The University of Sheffield

Mappin Street, Sheffield S1 3JD, United Kingdom 